\title{
On Effective Birkhoff's Ergodic Theorem for Computable Actions of Amenable Groups
}

\author{
Nikita Moriakov ${ }^{1,2}$ (D)
}

Abstract We introduce computable actions of computable groups and prove the following versions of effective Birkhoff's ergodic theorem. Let $\Gamma$ be a computable amenable group, then there always exists a canonically computable tempered twosided Følner sequence $\left(F_{n}\right)_{n \geq 1}$ in $\Gamma$. For a computable, measure-preserving, ergodic action of $\Gamma$ on a Cantor space $\{0,1\}^{\mathbb{N}}$ endowed with a computable probability measure $\mu$, it is shown that for every bounded lower semicomputable function $f$ on $\{0,1\}^{\mathbb{N}}$ and for every Martin-Löf random $\omega \in\{0,1\}^{\mathbb{N}}$ the equality

$$
\lim _{n \rightarrow \infty} \frac{1}{\left|F_{n}\right|} \sum_{g \in F_{n}} f(g \cdot \omega)=\int f d \mu
$$

holds, where the averages are taken with respect to a canonically computable tempered two-sided Følner sequence $\left(F_{n}\right)_{n \geq 1}$. We also prove the same identity for all lower semicomputable $f$ 's in the special case when $\Gamma$ is a computable group of polynomial growth and $F_{n}:=\mathrm{B}(n)$ is the Følner sequence of balls around the neutral element of $\Gamma$.

Keywords Effective ergodic theorems - Computable actions of groups

Nikita Moriakov

N.Moriakov@tudelft.nl;nm@dxdy.ru

1 Technische Universiteit Delft, Delft, Netherlands

2 Present address: ABN AMRO, Amsterdam, Netherlands 


\section{Introduction}

A classical ergodic theorem of Birkhoff asserts that, if $\varphi: X \rightarrow X$ is an ergodic measure-preserving transformation on a probability space $(X, \mu)$, then for every $f \in$ $\mathrm{L}^{1}(X)$ we have

$$
\lim _{n \rightarrow \infty} \frac{1}{n} \sum_{i=1}^{n} f\left(\varphi^{i} x\right)=\int f d \mu
$$

for $\mu$-a.e. $x \in X$. We refer, e.g., to [4, Chapter 11] for the proof. A celebrated result of Lindenstrauss [9] gives a generalization of Birkhoff's ergodic theorem for measurepreserving actions of amenable groups and ergodic averages, taken along tempered Følner sequences.

One may also wonder if the averages in (1.1) converge for every Martin-Löf random $x$ and every computable $f$. An affirmative answer was given by V'yugin in [14] for computable $f$ 's. Later, it was proved in [2] that the ergodic averages in (1.1) converge for every lower semi-computable $f$ and every Martin-Löf random $x$.

In so far, the effective ergodic theorems have only been proved for actions of $\mathbb{Z}$, and it is a natural question if one can generalize effective Birkhoff's ergodic theorem for measure-preserving actions of more general groups (for instance, the groups $\mathbb{Z}^{d}$, groups of polynomial growth and so on). However, one must first define computable actions of groups appropriately. In this article we define computable actions of computable groups in a natural way in Section 2.5, which agrees with the "classical' definition in the case of $\mathbb{Z}$-actions, and obtain the following generalizations of the results from [2]. First of all, we derive a generalization of Kučera's theorem in Section 3.1, which is the main technical tool of the article.

Theorem Let $\Gamma$ be a computable amenable group and $\left(\{0,1\}^{\mathbb{N}}, \mu, \Gamma\right)$ be a computable ergodic $\Gamma$-system. Let $U \subset\{0,1\}^{\mathbb{N}}$ be an effectively open subset such that $\mu(U)<1$. Let

$$
U^{*}:=\bigcap_{g \in \Gamma} g^{-1} U
$$

be the set of all points $\omega \in\{0,1\}^{\mathbb{N}}$ whose orbit remains in $U$. Then $U^{*}$ is an effectively null set.

Using this generalization of Kučera's theorem and the results of Lindenstrauss, we derive the first main theorem in Section 3.2. To simplify the notation, we denote the averages by $\mathbb{E}_{g \in F}:=\frac{1}{|F|} \sum_{g \in F}$.

Theorem Let $\Gamma$ be a computable amenable group with a canonically computable tempered two-sided $F \phi$ lner sequence $\left(F_{n}\right)_{n \geq 1}$. Suppose that $\left(\{0,1\}^{\mathbb{N}}, \mu, \Gamma\right)$ is a computable ergodic $\Gamma$-system. For every bounded lower semicomputable $f$ and for every Martin-Löf random $\omega \in\{0,1\}^{\mathbb{N}}$ the equality

$$
\lim _{n \rightarrow \infty} \mathbb{E}_{g \in F_{n}} f(g \cdot \omega)=\int f d \mu
$$

holds. 
In a special case, when $\Gamma$ is a computable group of polynomial growth, we are able to remove the boundedness assumption on $f$ and prove the following version of effective Birkhoff's ergodic theorem.

Theorem Let $\Gamma$ be a computable group of polynomial growth with the Folner sequence of balls around $\mathrm{e} \in \Gamma$ given by

$$
F_{n}:=\{g \in \Gamma:\|g\| \leq n\} \quad \text { for } n \geq 1 .
$$

Suppose that $\left(\{0,1\}^{\mathbb{N}}, \mu, \Gamma\right)$ is a computable ergodic $\Gamma$-system. For every lower semicomputable $f$ and for every Martin-Löf random $\omega \in\{0,1\}^{\mathbb{N}}$ the equality

$$
\lim _{n \rightarrow \infty} \mathbb{E}_{g \in F_{n}} f(g \cdot \omega)=\int f d \mu
$$

holds.

\section{Preliminaries}

\subsection{Computable Amenable Groups}

In this section we will remind the reader of the classical notion of amenability and state some results from ergodic theory of amenable group actions. We stress that all the groups that we consider are discrete and countably infinite.

Let $\Gamma$ be a group with the counting measure $|\cdot|$. A sequence of finite subsets $\left(F_{n}\right)_{n \geq 1}$ of $\Gamma$ is called

1) a left Følner sequence (resp. right Følner sequence) if for every $g \in \Gamma$ one has

$$
\frac{\left|F_{n} \Delta g F_{n}\right|}{\left|F_{n}\right|} \rightarrow 0 \quad\left(\text { resp. } \frac{\left|F_{n} \Delta F_{n} g\right|}{\left|F_{n}\right|} \rightarrow 0\right)
$$

2) a (C-)tempered sequence if there is a constant $C$ such that for every $j$ one has

$$
\left|\bigcup_{i<j} F_{i}^{-1} F_{j}\right|<C\left|F_{j}\right|
$$

A group is called amenable if it has a left Følner sequence. A sequence of finite subsets $\left(F_{n}\right)_{n \geq 1}$ of $\Gamma$ is called a two-sided Følner sequence if it is a left and a right Følner sequence simultaneously.

We refer the reader, e.g., to [13] for the standard notions of a computable function and a computable/enumerable set, which will appear in this article. A sequence of finite subsets $\left(F_{n}\right)_{n \geq 1}$ of $\mathbb{N}$ is called canonically computable if there is an algorithm that, given $n$, prints the set $F_{n}$ and halts. Formally speaking, for a finite set $A=$ $\left\{x_{1}, x_{2}, \ldots, x_{k}\right\} \subset \mathbb{N}$, we call the number $\mathrm{I}(A):=\sum_{i=1}^{k} 2^{x_{i}}$ the canonical index of 
$A$. Hence a sequence $\left(F_{n}\right)_{n \geq 1}$ of finite subsets of $\mathbb{N}$ is canonically computable if and only if the (total) function $n \mapsto \mathrm{I}\left(F_{n}\right)$ is computable.

A group $\Gamma$ with the composition operation $\circ$ is called a computable group if, as a set, $\Gamma$ is a computable subset of $\mathbb{N}$ and the total function $\circ: \Gamma \times \Gamma \rightarrow \Gamma$ is computable. It is easy to show that in a computable group $\Gamma$ the inversion operation $g \mapsto g^{-1}$ is a total computable function. We refer the reader to [12] for more details.

Any discrete amenable group $\Gamma$ admits a two-sided Følner sequence. Furthermore, if the group is computable, then there exists a canonically computable two-sided Følner sequence. To prove that we will need the following result.

Lemma 2.1 Given a discrete amenable group $\Gamma$, for any finite symmetric set $K \subset \Gamma$ such that $\mathrm{e} \in \Gamma$ and any $\varepsilon>0$ there exists a finite subset $F \subset \Gamma$ such that

$$
|K F K|-|F| \leq \varepsilon|F|
$$

We refer the reader to [10, I.§1, Proposition 2] for the proof.

Lemma 2.2 Let $\Gamma$ be a computable amenable group. Then there exists a canonically computable two-sided Folner sequence $\left(F_{n}\right)_{n \geq 1}$.

Proof First of all, observe that given $K \subset \Gamma, \varepsilon>0$ as in Lemma 2.1 and a finite set $F \subset \Gamma$ satisfying (2.1), we have

$$
\frac{|g F \backslash F|}{|F|} \leq \varepsilon
$$

and

$$
\frac{|F g \backslash F|}{|F|} \leq \varepsilon
$$

for all $g \in K$. Let $K_{n}$ be the finite set of the first $n$ elements of the computable group $\Gamma$. Then, for every $n=1,2, \ldots$ we apply Lemma 2.1 to the set $K_{n} \cup K_{n}^{-1} \cup\{\mathrm{e}\}$ and $\varepsilon_{n}:=1 / n$ and find the finite set $F_{n}$ with the smallest canonical index $\mathrm{I}\left(F_{n}\right)$ satisfying (2.1). It is easy to see that $\left(F_{n}\right)_{n \geq 1}$ is indeed a two-sided Følner sequence.

Every Følner sequence has a tempered Følner subsequence. Furthermore, the construction of a tempered Følner subsequence from a given canonically computable Følner sequence is 'algorithmic'. The proof is essentially contained in [9, Proposition 1.4], but we provide it for reader's convenience below.

Proposition 2.1 Let $\left(F_{n}\right)_{n \geq 1}$ be a canonically computable Folner sequence in a computable group $\Gamma$. Then there is a computable function $i \mapsto n_{i}$ s.t. the subsequence $\left(F_{n_{i}}\right)_{i \geq 1}$ is a canonically computable tempered $F \phi l n e r$ subsequence. 
Proof We define $n_{i}$ inductively as follows. Let $n_{1}:=1$. If $n_{1}, \ldots, n_{i}$ have been determined, we set $\widetilde{F}_{i}:=\bigcup_{j \leq i} F_{n_{j}}$. Take for $n_{i+1}$ the first integer greater than $i+1$ such that

$$
\left|F_{n_{i+1}} \triangle \widetilde{F}_{i}^{-1} F_{n_{i+1}}\right| \leq \frac{1}{\left|\widetilde{F}_{i}\right|}
$$

The function $i \mapsto n_{i}$ is total computable. It follows that

$$
\left|\bigcup_{j \leq i} F_{n_{j}}^{-1} F_{n_{i+1}}\right| \leq 2\left|F_{n_{i+1}}\right|
$$

hence the sequence $\left(F_{n_{i}}\right)_{i \geq 1}$ is 2-tempered. Since the Følner sequence $\left(F_{n}\right)_{n \geq 1}$ is canonically computable and the function $i \mapsto n_{i}$ is computable, the Følner sequence $\left(F_{n_{i}}\right)_{i \geq 1}$ is canonically computable and tempered.

Let us state an immediate corollary.

Corollary 2.1 Let $\Gamma$ be a computable amenable group. Then there exists a canonically computable, tempered two-sided Folner sequence $\left(F_{n}\right)_{n \geq 1}$ in $\Gamma$.

The following result tells us that the lim sup of averages of bounded functions on a group with a right Følner sequence is translation-invariant.

Lemma 2.3 (Limsup invariance) Let $\Gamma$ be a discrete group with a right Folner sequence $\left(F_{n}\right)_{n \geq 1}$ and $f \in \ell^{\infty}(\Gamma, \mathbb{R})$ be a bounded function on $\Gamma$. Then

$$
\limsup _{n \rightarrow \infty} \mathbb{E}_{g \in F_{n}} f(g)=\limsup _{n \rightarrow \infty} \mathbb{E}_{g \in F_{n}} f(g h) .
$$

Proof A direct computation shows that for all $n \geq 1$

$$
\frac{1}{\left|F_{n}\right|}\left|\sum_{g \in F_{n}} f(g)-\sum_{g \in F_{n} h} f(g)\right| \leq \frac{\left|F_{n} \Delta F_{n} h\right| \cdot\|f\|_{\infty}}{\left|F_{n}\right|},
$$

and the statement of the lemma follows since $\left(F_{n}\right)_{n \geq 1}$ is a right Følner sequence.

Remark 2.1 The statement of Lemma 2.3 does not hold for general amenable groups and unbounded nonnegative functions. As a counterexample, take $\Gamma:=\mathbb{Z}$ with the tempered two-sided Følner sequence

$$
F_{n}:=\left[-2^{n}, \ldots, 2^{n}\right] \quad \text { for } n \geq 1
$$

and define $f: \Gamma \rightarrow \mathbb{N}$ to be zero everywhere, except for points of the form $2^{k}+1$, where we let

$$
f\left(2^{k}+1\right):=2^{k} \quad \text { for all } k \geq 0 .
$$

It is then easy to see that

$$
\limsup _{n \rightarrow \infty} \mathbb{E}_{g \in F_{n}} f(g) \neq \limsup _{n \rightarrow \infty} \mathbb{E}_{g \in F_{n}} f(g+1) .
$$


We will resolve this issue in the class of groups of polynomial growth in Lemma 2.5 in Section 2.2.

\subsection{Computable Groups of Polynomial Growth}

Let $\Gamma$ be a finitely generated discrete group and $\left\{\gamma_{1}, \ldots, \gamma_{k}\right\}$ be a fixed generating set. Each element $\gamma \in \Gamma$ can be written as a product $\gamma_{i_{1}}^{p_{1}} \gamma_{i_{2}}^{p_{2}} \ldots \gamma_{i_{l}}^{p_{l}}$ for some indices $i_{1}, i_{2}, \ldots, i_{l} \in\{1, \ldots, k\}$ and some integers $p_{1}, p_{2}, \ldots, p_{l} \in \mathbb{Z}$. We define the norm of an element $\gamma \in \Gamma$ by

$$
\|\gamma\|:=\inf \left\{\sum_{i=1}^{l}\left|p_{i}\right|: \gamma=\gamma_{i_{1}}^{p_{1}} \gamma_{i_{2}}^{p_{2}} \ldots \gamma_{i_{l}}^{p_{l}}\right\},
$$

where the infinum is taken over all representations of $\gamma$ as a product of the generating elements. The norm $\|\cdot\|$ on $\Gamma$ can, in general, depend on the generating set, but it is easy to show [3, Corollary 6.4.2] that two different generating sets produce equivalent norms. We will always say what generating set is used in the definition of a norm, but we will omit an explicit reference to the generating set later on. Using this norm, we define unit balls in $\Gamma$ as

$$
\mathrm{B}(n):=\{g \in \Gamma:\|g\| \leq n\} \text { for all } n \geq 0 .
$$

We say that the group $\Gamma$ is of polynomial growth if there are constants $C, d>0$ such that for all $n \geq 1$ we have

$$
|\mathrm{B}(n)| \leq C n^{d}
$$

Example 2.1 Consider the group $\mathbb{Z}^{d}$ for $d \in \mathbb{N}$ and let $\gamma_{1}, \ldots, \gamma_{d} \in \mathbb{Z}^{d}$ be the standard basis elements of $\mathbb{Z}^{d}$. That is, $\gamma_{i}$ is defined by

$$
\gamma_{i}(j):=\delta_{i}^{j} \quad(j=1, \ldots, d)
$$

for all $i=1, \ldots, d$. We consider the generating set given by elements $\sum_{k \in I}(-1)^{\varepsilon_{k}} \gamma_{k}$ for all subsets $I \subseteq[1, d]$ and all functions $\varepsilon$. $\in\{0,1\}^{I}$. Then it is easy to see by induction on dimension that $\mathrm{B}(n)=[-n, \ldots, n]^{d}$, hence

$$
|\mathrm{B}(n)|=(2 n+1)^{d} \quad \text { for all } n \in \mathbb{N}
$$

with respect to this generating set, i.e., $\mathbb{Z}^{d}$ is a group of polynomial growth.

Let $d \in \mathbb{Z}_{\geq 0}$. We say that the group $\Gamma$ has polynomial growth of degree $d$ if there is a constant $C>0$ such that

$$
\frac{1}{C} n^{d} \leq|\mathrm{B}(n)| \leq C n^{d} \quad \text { for all } n \in \mathbb{N} .
$$

It was shown in [1] that, if $\Gamma$ is a finitely generated nilpotent group, then $\Gamma$ has polynomial growth of some degree $d \in \mathbb{Z} p$. Furthermore, one can show [3, Proposition 6.6.6] that if $\Gamma$ is a group and $\Gamma^{\prime} \leq \Gamma$ is a finite index, finitely generated nilpotent subgroup, having polynomial growth of degree $d \in \mathbb{Z} p$, then the group $\Gamma$ has polynomial growth of degree $d$. The converse is true as well: it was proved in [7] that, if 
$\Gamma$ is a group of polynomial growth, then there exists a finite index, finitely generated nilpotent subgroup $\Gamma^{\prime} \leq \Gamma$. It follows that if $\Gamma$ is a group of polynomial growth, then there is a constant $C>0$ and an integer $d \in \mathbb{Z}_{\geq 0}$, called the degree of polynomial growth, such that

$$
\frac{1}{C} n^{d} \leq|\mathrm{B}(n)| \leq C n^{d} \quad \text { for all } n \in \mathbb{N} .
$$

An even stronger result was obtained in [11], where it is shown that, if $\Gamma$ is a group of polynomial growth of degree $d \in \mathbb{Z}_{\geq 0}$, then the limit

$$
c_{\Gamma}:=\lim _{n \rightarrow \infty} \frac{|\mathrm{B}(n)|}{n^{d}}
$$

exists.

Lemma 2.4 Let $\Gamma$ be a group of polynomial growth. Then $(\mathrm{B}(n))_{n \geq 1}$ is a tempered two-sided Folner sequence in $\Gamma$.

Proof We want to show that for every $g \in \Gamma$

$$
\lim _{n \rightarrow \infty} \frac{|g \mathrm{~B}(n) \triangle \mathrm{B}(n)|}{|\mathrm{B}(n)|}=0 .
$$

Let $m:=\|g\| \in \mathbb{Z}_{\geq 0}$. Then $g \mathrm{~B}(n) \subseteq \mathrm{B}(n+m)$ and $g^{-1} \mathrm{~B}(n) \subseteq \mathrm{B}(n+m)$, hence

$$
\frac{|g \mathrm{~B}(n) \Delta \mathrm{B}(n)|}{|\mathrm{B}(n)|} \leq \frac{2|\mathrm{~B}(n+m) \backslash \mathrm{B}(n)|}{|\mathrm{B}|(n)} \leq \frac{2(|\mathrm{~B}(n+m)|-|\mathrm{B}(n)|)}{|\mathrm{B}(n)|} \rightarrow 0,
$$

where we use the existence of the limit in (2.2). Similarly, we use the relation $\mathrm{B}(n) g \subseteq \mathrm{B}(n+m)$ to show that $(\mathrm{B}(n))_{n \geq 1}$ is a right Følner sequence. The sequence $(\mathrm{B}(n))_{n \geq 1}$ is tempered, since

$$
|\mathrm{B}(n-1) \cdot \mathrm{B}(n)| \leq|\mathrm{B}(2 n)| \leq C^{2} 2^{d}|\mathrm{~B}(n)|
$$

for all $n \geq 1$.

As promised in Remark 2.1, we prove now that the lim sup of averages of arbitrary nonnegative functions on a group of polynomial growth $\Gamma$ is translation invariant.

Lemma 2.5 (Limsup invariance) Let $\Gamma$ be a group of polynomial growth and define the Folner sequence of balls around e $\in \Gamma$ by

$$
F_{n}:=\{g \in \Gamma:\|g\| \leq n\} \quad \text { for } n \geq 1 .
$$

Let $f: \Gamma \rightarrow \mathbb{R}_{\geq 0}$ be a nonnegative function on $\Gamma$. Then

$$
\limsup _{n \rightarrow \infty} \mathbb{E}_{g \in F_{n}} f(g)=\limsup _{n \rightarrow \infty} \mathbb{E}_{g \in F_{n}} f(g h)
$$

for all $h \in \Gamma$. 
Proof Let $S \subset \Gamma$ be the finite generating set, which is used in the definition of the norm $\|\cdot\|$ on $\Gamma$. Since the statement of the lemma is 'symmetric' and since the set $S$ generates $\Gamma$, it suffices to prove that

$$
\limsup _{n \rightarrow \infty} \mathbb{E}_{g \in F_{n}} f(g) \geq \limsup _{n \rightarrow \infty} \mathbb{E}_{g \in F_{n}} f(g h)
$$

for all $h \in S \cup S^{-1}$. We fix an element $h \in S \cup S^{-1}$. It is clear that $F_{n} h \subseteq F_{n+1}$, hence

$$
\limsup _{n \rightarrow \infty} \mathbb{E}_{g \in F_{n}} f(g h) \leq \limsup _{n \rightarrow \infty} \frac{1}{\left|F_{n}\right|} \sum_{g \in F_{n+1}} f(g) .
$$

But

$$
\limsup _{n \rightarrow \infty} \frac{1}{\left|F_{n}\right|} \sum_{g \in F_{n+1}} f(g)=\limsup _{n \rightarrow \infty} \frac{\left|F_{n+1}\right|}{\left|F_{n}\right|} \cdot \mathbb{E}_{g \in F_{n+1}} f(g)
$$

and $\left|F_{n+1}\right| /\left|F_{n}\right| \rightarrow 1$ as $n \rightarrow \infty$, which implies that

$$
\limsup _{n \rightarrow \infty} \frac{1}{\left|F_{n}\right|} \sum_{g \in F_{n+1}} f(g)=\limsup _{n \rightarrow \infty} \mathbb{E}_{g \in F_{n}} f(g),
$$

and the proof is complete.

Whenever discussing computable groups of polynomial growth, we will always assume that the generating set is known and fixed. We state the following lemma.

Lemma 2.6 Let $\Gamma$ be a finitely generated group with a distinguished set of generators $\left\{\gamma_{1}, \ldots, \gamma_{k}\right\}$. Then the following assertions hold:

(a) The sequence of balls $(\mathrm{B}(n))_{n \geq 1}$ is a canonically computable sequence of finite sets;

(b) The growth function $n \mapsto|\mathrm{B}(n)|, \mathbb{Z}_{\geq 0} \rightarrow \mathbb{N}$ is a total computable function;

(c) The norm $\|\cdot\|: \Gamma \rightarrow \mathbb{Z}_{\geq 0}$ is a total computable function.

The proof of the lemma is straightforward.

\subsection{Ergodic Theory}

Let $\mathrm{X}=(X, \mathcal{B}, \mu)$ be a probability space. A measurable transformation $\varphi: X \rightarrow X$ is called measure-preserving if

$$
\mu\left(\varphi^{-1} A\right)=\mu(A) \quad \text { for all } A \in \mathcal{B} .
$$

A measure-preserving transformation $\varphi: X \rightarrow X$ is called an automorphism if there exists a measure-preserving transformation $\psi: X \rightarrow X$ such that

$$
\varphi \circ \psi=\psi \circ \varphi=\operatorname{id}_{X} \quad \mu-\text { a.e. }
$$

We denote by $\operatorname{Aut}(\mathrm{X})$ the group of all automorphisms of the probability space $\mathrm{X}$. Given a discrete group $\Gamma$, a measure-preserving $\Gamma$-system ${ }^{1}$ is a probability space

\footnotetext{
${ }^{1}$ To simplify the notation, the shorter term ' $\Gamma$-system' will also be used.
} 
$\mathrm{X}=(X, \mathcal{B}, \mu)$, endowed with an action of $\Gamma$ on $X$ by automorphisms from $\operatorname{Aut}(\mathrm{X})$. We denote a measure-preserving $\Gamma$-system on a probability space $(X, \mathcal{B}, \mu)$ by a triple $(X, \mu, \Gamma)$ and we write $g \cdot x$, where $g \in \Gamma, x \in X$, to denote the corresponding action of $\Gamma$ on elements of $X$.

Let $\mathbf{X}=(X, \mu, \Gamma)$ be a measure-preserving $\Gamma$-system on a probability space $(X, \mathcal{B}, \mu)$. We say that $\mathbf{X}$ is ergodic (or that the measure $\mu$ on $X$ is ergodic) if, for all $A \in \mathcal{B}$, the condition

$$
\mu\left(\gamma^{-1} A \triangle A\right)=0 \quad \text { for all } \gamma \in \Gamma
$$

implies that $\mu(A)=0$ or $\mu(A)=1$. That is, $\mathbf{X}$ is ergodic if only the trivial sets are essentially invariant under $\Gamma$.

The simplest ergodic theorem for amenable group actions is the mean ergodic theorem, which we state below. For the proof we refer the reader to [6, Theorem 3.33].

Theorem 2.1 Let $(X, \mu, \Gamma)$ be a measure-preserving, ergodic $\Gamma$-system, where the group $\Gamma$ is amenable and $\left(F_{n}\right)_{n \geq 1}$ is a left Folner sequence. Then for every $f \in$ $\mathrm{L}^{2}(X)$ we have

$$
\lim _{n \rightarrow \infty} \mathbb{E}_{g \in F_{n}} f \circ g=\int f d \mu,
$$

where the convergence is understood in $\mathrm{L}^{2}(X)$-sense.

Pointwise convergence of ergodic averages is much more tricky, in particular, pointwise ergodic averages do not necessarily converge, unless the Følner sequence satisfies some additional assumptions. The following important theorem was proved by E. Lindenstrauss in [9]. ${ }^{2}$

Theorem 2.2 Let $\mathbf{X}=(\mathrm{X}, \mu, \Gamma)$ be an ergodic measure-preserving $\Gamma$-system, where the group $\Gamma$ is amenable and $\left(F_{n}\right)_{n \geq 1}$ is a tempered left Folner sequence. Then for every $f \in \mathrm{L}^{1}(X)$

$$
\lim _{n \rightarrow \infty} \mathbb{E}_{g \in F_{n}} f(g \cdot x)=\int f d \mu
$$

for $\mu$-a.e. $x \in X$.

\subsection{Computability on Cantor Space and Martin-Löf Randomness}

In this section we remind the reader some standard notions of computability on Cantor space. All of these notions have analogs on computable metric spaces as well, and we refer to $[5,8]$ for the details.

\footnotetext{
${ }^{2}$ In fact, a more general statement is proved there, but we only need the ergodic case in this work.
} 
Throughout the article we fix some enumeration of $\mathbb{Q}=\left\{q_{1}, q_{2}, q_{2}, \ldots\right\}$. We use the standard notions of a computable real number and of a lower/upper semicomputable real number. A sequence of real numbers $\left(a_{n}\right)_{n \geq 1}$ is called computable uniformly in $n$ if there exists an algorithm $A: \mathbb{N} \times \mathbb{N} \rightarrow \mathbb{Q}$ such that

$$
\left|A(n, i)-a_{n}\right|<2^{-i} \text { for all } n, i \geq 1 .
$$

We fix the lexicographic enumeration

$$
\{0,1\}^{*}=\left\{\mathrm{w}_{1}, \mathrm{w}_{2}, \ldots\right\}
$$

of the set of all finite binary words, where, firstly, appears the block of all words of length 1 ordered lexicographically, then the block of all words of length 2 ordered lexicographically and so on. Let

$$
[\mathrm{w}]:=\left\{\omega: \omega=\mathrm{w} \omega^{\prime} \text { for some } \omega^{\prime} \in\{0,1\}^{\mathbb{N}}\right\} \subset\{0,1\}^{\mathbb{N}}
$$

be the cylinder set of all words that begin with a finite word $w \in\{0,1\}^{*}$. A set $U \subseteq\{0,1\}^{\mathbb{N}}$ is called effectively open if there is a recursively enumerable subset $E \subseteq \mathbb{N}$ such that

$$
U=\bigcup_{i \in E}\left[\mathrm{w}_{i}\right] .
$$

A sequence $\left(U_{n}\right)_{n \geq 1}$ of sets is called a uniformly effectively open sequence of sets if there is a recursively enumerable set $E \subseteq \mathbb{N} \times \mathbb{N}$ such that

$$
U_{i}=\bigcup_{(i, j) \in E}\left[\mathrm{w}_{j}\right] \quad \text { for all } i \geq 1 .
$$

A mapping $\varphi:\{0,1\}^{\mathbb{N}} \rightarrow\{0,1\}^{\mathbb{N}}$ is called computable if $\left(\varphi^{-1}\left(\left[\mathrm{w}_{i}\right]\right)\right)_{i \geq 1}$ is uniformly effectively open, that is, there is a recursively enumerable set $E_{\varphi} \subseteq \mathbb{N} \times \mathbb{N}$ such that

$$
\varphi^{-1}\left(\left[\mathrm{w}_{i}\right]\right)=\bigcup_{(i, j) \in E_{\varphi}}\left[\mathrm{w}_{j}\right] \quad \text { for all } i \geq 1 .
$$

A function $f:\{0,1\}^{\mathbb{N}} \rightarrow \mathbb{R}_{\geq 0}$ is called lower semicomputable if the sequence of sets $\left(f^{-1}\left(\left(q_{n},+\infty\right)\right)\right)_{n \geq 1}$ is uniformly effectively open.

Let $\mu$ be a Borel probability measure on $\{0,1\}^{\mathbb{N}}$. We say that $\mu$ is a computable measure $^{3}$ if

$$
\mu\left(\left[\mathrm{w}_{i_{1}}\right] \cup\left[\mathrm{w}_{i_{2}}\right] \cup \cdots \cup\left[\mathrm{w}_{i_{k}}\right]\right)
$$

is computable uniformly in $i_{1}, \ldots, i_{k} \geq 1$.

Suppose that $\mu$ is a computable probability measure on $\{0,1\}^{\mathbb{N}}$. A Martin-Löf $\mu$-test is a uniformly effectively open sequence of sets $\left(U_{n}\right)_{n \geq 1}$ such that

$$
\mu\left(U_{n}\right)<2^{-n} \quad \text { for all } n \geq 1 \text {. }
$$

\footnotetext{
${ }^{3}$ One can also restrict to the measures $\mu([\mathrm{w}])$ of the cylinder sets and require uniform computability of these only.
} 
Any subset of $\bigcap_{n \geq 1} U_{n}$ is called an effectively $\mu$-null set. A point $\omega \in\{0,1\}^{\mathbb{N}}$ is called

Martin-Löf random if it is not contained in any effectively $\mu$-null set.

\subsection{Computable Dynamical Systems}

Now, let $\Gamma \subseteq \mathbb{N}$ be a computable group, which acts on $\{0,1\}^{\mathbb{N}}$ by homeomorphisms. We say that the action of $\Gamma$ is computable if there is a recursively enumerable subset $E \subseteq \Gamma \times \mathbb{N} \times \mathbb{N}$ such that

$$
\gamma^{-1}\left(\left[\mathrm{w}_{i}\right]\right)=\bigcup_{(\gamma, i, j) \in E}\left[\mathrm{w}_{j}\right] \quad \text { for all } i \geq 1, \gamma \in \Gamma .
$$

In general, checking the computability of the action of a computable group $\Gamma$ on $\{0,1\}^{\mathbb{N}}$ can be trickier than checking computability of a single transformation. Imagine a $\mathbb{Z}$-action on $\{0,1\}^{\mathbb{N}}$ with the generating element $\varphi \in \mathbb{Z}$. Can it happen that both $\varphi$ and $\varphi^{-1}$ act by computable transformations on $\{0,1\}^{\mathbb{N}}$, while the action of $\mathbb{Z}$ on $\{0,1\}^{\mathbb{N}}$ is not computable? Fortunately, the answer is 'no': the following lemma tells us that for an action of a computable finitely generated group it suffices to check computability of transformations in a finite symmetric generating set to guarantee the computability of the action. The lemma also shows that the terminology of computable group actions which we suggest in this article is compatible with the classical case, when there is only one computable transformation.

Lemma 2.7 Let $\Gamma$ be a finitely generated computable group with a finite symmetric generating set $S \subset \Gamma$. Suppose that $\Gamma$ acts on $\{0,1\}^{\mathbb{N}}$ by homeomorphisms, and, furthermore, that for each $\gamma \in S$ the transformation

$$
\gamma:\{0,1\}^{\mathbb{N}} \rightarrow\{0,1\}^{\mathbb{N}}
$$

is computable. Then the action of $\Gamma$ on $\{0,1\}^{\mathbb{N}}$ is computable.

Proof Given a fixed finite symmetric generating set $S=\left\{\gamma_{1}, \gamma_{2}, \ldots, \gamma_{N}\right\}$, we will denote by $\mathrm{B}(n)$ the corresponding balls around the neutral element $\mathrm{e} \in \Gamma$ with respect to the norm determined by $S$. Since $\gamma_{1}, \gamma_{2}, \ldots, \gamma_{N}$ are computable endomorphisms of $\{0,1\}^{\mathbb{N}}$, there are recursively enumerable subsets $E_{1}, E_{2}, \ldots, E_{N}$ such that

$$
\gamma_{k}^{-1}\left(\left[\mathrm{w}_{i}\right]\right)=\bigcup_{(i, j) \in E_{k}}\left[\mathrm{w}_{j}\right] \quad \text { for all } k=1, \ldots, N, i \geq 1 .
$$

We will describe an algorithm, which enumerates the set $E$. At stage $n$, the algorithm first computes the finite set $\mathrm{B}(n) \subset \Gamma$ by computing all products of the elements of $S$ of length at most $n$. For each word

$$
\gamma_{i_{1}} \gamma_{i_{2}} \ldots \gamma_{i_{k}}=\gamma \in \mathrm{B}(n)
$$


we have for all $i \geq 1$

$$
\begin{aligned}
\gamma^{-1}\left(\left[\mathrm{w}_{i}\right]\right) & =\gamma_{i_{k}}^{-1} \gamma_{i_{k-1}}^{-1} \ldots \gamma_{i_{1}}^{-1}\left(\left[\mathrm{w}_{i}\right]\right)=\gamma_{i_{k}}^{-1} \gamma_{i_{k-1}}^{-1} \ldots \gamma_{i_{2}}^{-1} \bigcup_{\left(i, j_{1}\right) \in E_{i_{1}}}\left[\mathrm{w}_{j_{1}}\right] \\
& =\gamma_{i_{k}}^{-1} \gamma_{i_{k-1}}^{-1} \ldots \gamma_{i_{3}}^{-1} \bigcup_{\left(i, j_{1}\right) \in E_{i_{1}}} \bigcup_{\left(j_{1}, j_{2}\right) \in E_{i_{2}}}\left[\mathrm{w}_{j_{2}}\right] \\
& =\bigcup_{\left(i, j_{1}\right) \in E_{i_{1}}\left(j_{1}, j_{2}\right) \in E_{i_{2}}} \bigcup_{\left(j_{2}, j_{3}\right) \in E_{i_{3}}} \cdots \bigcup_{\left(j_{k-1}, j_{k}\right) \in E_{i_{k}}}\left[\mathrm{w}_{j_{k}}\right] .
\end{aligned}
$$

We compute the first $n$ pairs $\left(i, j_{1}\right) \in E_{i_{1}}$, for each of these pairs we compute the first $n$ pairs $\left(j_{1}, j_{2}\right) \in E_{i_{2}}$ and so on up to the first $n$ pairs $\left(j_{k-1}, j_{k}\right) \in E_{i_{k}}$ (where $j_{k-1}$ comes from the one but the last step). The algorithm prints all resulting triples $\left(\gamma, i, j_{k}\right)$, and proceeds to the next word (or the next stage, if all words at the current stage have been exhausted).

Since, at each stage $n$, we look through all products of length at most $n$, it is easy to see that

$$
\gamma^{-1}\left(\left[\mathrm{w}_{i}\right]\right)=\bigcup_{(\gamma, i, j) \in E}\left[\mathrm{w}_{j}\right]
$$

for all $i \geq 1$, and, furthermore, the set $E$ is recursively enumerable.

A computable Cantor $\Gamma$-system ${ }^{4}$ is a triple $\left(\{0,1\}^{\mathbb{N}}, \mu, \Gamma\right)$, where $\mu$ is a computable measure on $\{0,1\}^{\mathbb{N}}$ and $\Gamma$ acts computably on $\{0,1\}^{\mathbb{N}}$ by measure-preserving transformations.

Remark 2.2 The notion of a computable action of a computable group which we suggest directly translates to arbitrary computable metric spaces. Furthermore, Lemma 2.7 remains valid in the more general setting.

To finish this section, we give a basic example of a computable Cantor system.

Example 2.2 Consider the group $\mathbb{Z}^{d}$ and let $\iota: \mathbb{Z}^{d} \rightarrow \mathbb{N}$ be a computable bijection s.t. $\mathbb{Z}^{d}$ is a computable group when viewed as a set $\iota\left(\mathbb{Z}^{d}\right)=\mathbb{N}$. $\mathbb{Z}^{d}$ acts on the compact space $\{0,1\}^{\mathbb{N}}=\{0,1\}^{l\left(\mathbb{Z}^{d}\right)}$ by shift transformation:

$$
(g \cdot \omega)(y)=\omega\left(l\left(g+l^{-1}(y)\right)\right) \text { for } g \in \mathbb{Z}^{d}, y \in \mathbb{N}, \omega \in\{0,1\}^{\mathbb{N}} .
$$

Fix a Bernoulli product measure on $\{0,1\}^{\mathbb{N}}$. Since the action of $\mathbb{Z}^{d}$ on $\{0,1\}^{\mathbb{N}}$ maps cylinder sets to cylinder sets with the same number of defining conditions, we deduce that this action is measure-preserving. It remains to show that the action is computable. Lemma 2.7 tells us that it is enough to show the computability of transformations $\gamma_{i}: \omega \mapsto \gamma_{i} \cdot \omega$ for some symmetric generating set $\gamma_{1}, \ldots, \gamma_{2 d}$ of $\mathbb{Z}^{d}$. Fix

\footnotetext{
${ }^{4}$ Or a computable $\Gamma$-system for short, since we only consider dynamical systems on Cantor space in this article.
} 
an arbitrary generator $\gamma$. We want to find recursively enumerable set $E_{\gamma} \subseteq \mathbb{N} \times \mathbb{N}$ such that

$$
\gamma^{-1}\left(\left[\mathrm{w}_{i}\right]\right)=\bigcup_{(i, j) \in E_{\gamma}}\left[\mathrm{w}_{j}\right] \quad \text { for all } i \geq 1 .
$$

Preimage of a cylinder set

$$
[\mathrm{w}]=\left\{x \in\{0,1\}^{\mathbb{N}}: x_{1}=\mathrm{w}_{1}, x_{2}=\mathrm{w}_{2}, \ldots, x_{k}=\mathrm{w}_{k}\right\}
$$

is a cylinder set

$$
\gamma^{-1}[\mathrm{w}]=\left\{x \in\{0,1\}^{\mathbb{N}}: x_{j_{1}}=\mathrm{w}_{1}, x_{j_{2}}=\mathrm{w}_{2}, \ldots, x_{j_{k}}=\mathrm{w}_{k}\right\},
$$

where the indexes $j_{1}, j_{2}, \ldots, j_{k}$ can be computed from the index of the word $\mathrm{w} \in$ $\{0,1\}^{*}$. Hence the set $E_{\gamma}$ can be obtained as follows. At stage $n \geq 1$, we test the first $n$ indexes $i$ and the first $n$ indexes $j$. For a given pair $(i, j)$, we check if the word $\mathrm{w}_{j}$ belongs to the cylinder set $\gamma^{-1}\left[\mathrm{w}_{i}\right]$. If it does, then the pair $(i, j)$ is added to $E_{\gamma}$.

\section{Effective Birkhoff's Theorem}

\subsection{Kučera's Theorem}

In this section we generalize Kučera's theorem for computable actions of amenable groups. In the proof we follow roughly the approach from [2], although the technical details do differ.

Theorem 3.1 Let $\Gamma$ be a computable amenable group and $\left(\{0,1\}^{\mathbb{N}}, \mu, \Gamma\right)$ be a computable ergodic $\Gamma$-system. Let $U \subset X$ be an effectively open subset such that $\mu(U)<1$. Let

$$
U^{*}:=\bigcap_{g \in \Gamma} g^{-1} U
$$

be the set of all points $\omega \in\{0,1\}^{\mathbb{N}}$ whose orbit remains in $U$. Then $U^{*}$ is an effectively null set.

Proof Let $\left(F_{n}\right)_{n \geq 1}$ be a canonically computable two-sided Følner sequence in $\Gamma$ and $\mu(U)<q<1$ be some fixed rational number. Let $\left(I_{i}\right)_{i \geq 1}$ be the basis of cylinder sets in $\left(\{0,1\}^{\mathbb{N}}, \mu\right)$. Let

$$
(i, k) \mapsto n(i, k), \quad \mathbb{N} \times \mathbb{N} \rightarrow \mathbb{N}
$$

be some total computable function, which will be chosen later, and define a computable function $m$ by

$$
m(i, k):=\left|F_{n(i, k)}\right| \quad \text { for } i, k \geq 1 .
$$


Since $U_{0}:=U$ is effectively open, there is a r.e. subset $E_{0} \subseteq \mathbb{N}$ such that $U_{0}=$ $\bigcup_{i \in E_{0}} I_{i}$ is a union of disjoint cylinder sets. Since the action of $\bar{\Gamma}$ is computable and since $\left(F_{n}\right)_{n \geq 1}$ is canonically computable, the sequence

$$
i \mapsto I_{i} \cap \bigcap_{g \in F_{n(i, 1)}} g^{-1}\left(U_{0}\right)
$$

is a uniformly effectively open sequence of sets. Let

$$
U_{1}:=\bigcup_{i \in E_{0}}\left(I_{i} \cap \bigcap_{g \in F_{n(i, 1)}} g^{-1}\left(U_{0}\right)\right),
$$

then, clearly, $U_{1} \subseteq U_{0}$ is an effectively open set and $U^{*} \subseteq U_{1}$. Since $U_{1}$ is an effectively open set, there is a r.e. subset $E_{1} \subseteq \mathbb{N}$ such that $U_{1}=\bigcup_{i \in E_{1}} I_{i}$ is a union of disjoint cylinder sets. Suppose that

$$
\mu\left(I_{i} \cap \bigcap_{g \in F_{n(i, 1)}} g^{-1}(U)\right)<q \mu\left(I_{i}\right)+q \cdot 2^{-i} \quad \text { for all } i \geq 1 .
$$

The cylinder sets $\left(I_{i}\right)_{i \in E_{0}}$ are pairwise disjoint, hence $\mu\left(U_{1}\right) \leq q \mu\left(U_{0}\right)+q$.

We want to apply the same procedure to $U_{1}$ and so on to obtain a sequence of uniformly open sets with almost exponentially decaying measure. So, in general, let $k \geq 1$ and suppose that $U_{k-1}=\bigcup_{i \in E_{k-1}} I_{i}$ is a disjoint union of cylinder sets for an r.e. subset $E_{k-1}$. We let

$$
\begin{aligned}
U_{k}:=\bigcup_{i \in E_{k-1}}\left(I_{i} \cap \bigcap_{g \in F_{n(i, k)}} g^{-1}\left(U_{k-1}\right)\right)=\bigcup_{i \in E_{k-1}}\left(I_{i} \cap \bigcap_{g \in F_{n(i, k)}} \bigcup_{j \in E_{k-1}} g^{-1}\left(I_{j}\right)\right) \\
=\bigcup_{i \in E_{k-1} j_{1}, \ldots, j_{m(i, k)} \in E_{k-1}}\left(I_{i} \cap \bigcap_{s=1}^{m(i, k)} g_{i, s}^{-1}\left(I_{j_{s}}\right)\right),
\end{aligned}
$$

where $g_{i, 1}, g_{i, 2}, \ldots, g_{i, m(i, k)}$ is the list of all distinct elements of $F_{n(i, k)}$. The sequence of sets

$$
\left(I_{i} \cap \bigcap_{s=1}^{m(i, k)} g_{i, s}^{-1}\left(I_{j_{s}}\right)\right)_{i, j_{1}, \ldots, j_{m(i, k)}}
$$

is uniformly effectively open, so it follows that $\left(U_{k}\right)_{k \geq 1}$ is uniformly effectively open. Clearly, $U^{*} \subseteq U_{k} \subseteq U_{k-1}$ for every $k \geq 1$. If we show that

$$
\mu\left(I_{i} \cap \bigcap_{g \in F_{n(i, k)}} g^{-1}\left(U_{k-1}\right)\right)<q \mu\left(I_{i}\right)+q^{k} \cdot 2^{-i} \quad \text { for all } i \geq 1
$$


then $\mu\left(U_{k}\right)<q \mu\left(U_{k-1}\right)+q^{k}$ for every $k$, and so $\mu\left(U_{k}\right)<(k+1) q^{k}$, which would imply that $U^{*}$ is an effectively null set. Observe that

$$
\begin{aligned}
& \mu\left(I_{i} \cap \bigcap_{g \in F_{n(i, k)}} g^{-1}\left(U_{k-1}\right)\right) \leq \min _{g \in F_{n(i, k)}} \mu\left(I_{i} \cap g^{-1}\left(U_{k-1}\right)\right) \\
& \leq \mathbb{E}_{g \in F_{n(i, k)}} \mu\left(I_{i} \cap g^{-1}\left(U_{k-1}\right)\right)=\mathbb{E}_{g \in F_{n(i, k)}} \mu\left(\left(g I_{i}\right) \cap U_{k-1}\right) \\
& =\int\left(\mathbb{E}_{g \in F_{n(i, k)}^{-1}} \mathbf{1}_{I_{i}}(g \cdot \omega)\right) \mathbf{1}_{U_{k-1}}(\omega) d \mu .
\end{aligned}
$$

If, for every $i, k \geq 1$, we find effectively a number $n(i, k)$ such that

$$
\left\|\mathbb{E}_{g \in F_{n(i, k)}^{-1}} \mathbf{1}_{I_{i}}(g \cdot \omega)-\mu\left(I_{i}\right)\right\|_{2}<q^{k} \cdot 2^{-i},
$$

then, due to Cauchy-Schwarz inequality, the computation above implies that

$$
\mu\left(I_{i} \cap \bigcap_{g \in F_{n(i, k)}} g^{-1}\left(U_{k-1}\right)\right) \leq q \mu\left(I_{i}\right)+q^{k} \cdot 2^{-i} .
$$

Mean ergodic theorem (Theorem 2.1) implies that a number $n(i, k)$ satisfying (3.4) always exists, since $\left(F_{n}^{-1}\right)_{n \geq 1}$ is a left Følner sequence. To find the number $n(i, k)$ effectively we argue as follows. 5

First, $\left(g I_{i}\right)_{g \in \Gamma, i \geq 1}$ is a uniformly effectively open sequence of sets by definition of computability of the action of $\Gamma$ on $\{0,1\}^{\mathbb{N}}$, so let $E \subseteq \Gamma \times \mathbb{N} \times \mathbb{N}$ be an r.e. subset such that

$$
g\left(I_{i}\right)=\bigcup_{(g, i, j) \in E} I_{j} \quad \text { for all } g \in \Gamma, i \geq 1
$$

We claim that there exists a uniformly effectively open sequence of sets $\left(\Delta_{g, i}^{k}\right)_{g, i, k}$, where each $\Delta_{g, i}^{k}$ is the union of the first $\left|\Delta_{g, i}^{k}\right|$ intervals in $g I_{i}$, such that the function $(g, i, k) \mapsto\left|\Delta_{g, i}^{k}\right|$ is total computable and that

$$
\mu\left(g I_{i} \backslash \Delta_{g, i}^{k}\right)<\frac{q^{2 k} \cdot 2^{-2 i}}{64} \quad \text { for all } g \in \Gamma \text { and } i, k \geq 1 \text {. }
$$

To do so, we use computability of the measure $\mu$ to find (uniformly in $i, k$ and effectively) a rational $d_{i}^{k}$ such that

$$
\left|\mu\left(I_{i}\right)-d_{i}^{k}\right|<\frac{q^{2 k} \cdot 2^{-2 i}}{256} \quad \text { for all } i, k \geq 1 .
$$

\footnotetext{
${ }^{5}$ It was pointed out by the reviewer that the rest of the proof can be shortened by noticing that the integral of a computable, bounded function with respect to a computable measure is computable.
} 
The set $\Delta_{g, i}^{k}$ is constructed as follows. Let $\Delta_{g, i}^{k}=\varnothing$. Take the first interval $I_{j_{1}}$ such that $\left(g, i, j_{1}\right) \in E$, add it to the collection $\Delta_{g, i}^{k}$ and compute its measure $\tilde{m}_{g, i}$ with precision $\frac{q^{2 k} \cdot 2^{-2 i}}{256}$. If

$$
\tilde{m}_{g, i}>d_{i}^{k}-\frac{q^{2 k} \cdot 2^{-2 i}}{128},
$$

then we are done. Otherwise, we add the next interval $I_{j_{2}}$ such that $\left(g, i, j_{2}\right) \in E$ to the collection $\Delta_{g, i}^{k}$, compute the measure $\tilde{m}_{g, i}$ of the union of intervals in $\Delta_{g, i}^{k}$ with precision $\frac{q^{2 k} \cdot 2^{-2 i}}{256}$ and check the condition (3.6) once again and so on. The algorithm eventually terminates, it is clear that it provides a uniformly effectively open sequence of sets $\left(\Delta_{g, i}^{k}\right)_{g, i, k}$, and a direct computation shows that condition (3.5) is satisfied as well.

The number $n(i, k)$ is defined as the smallest nonnegative integer such that

$$
\left\|\mathbb{E}_{g \in F_{n(i, k)}} \mathbf{1}_{\Delta_{g, i}^{k}}-d_{i}^{k}\right\|_{2}<\frac{q^{k} \cdot 2^{-i}}{2},
$$

where the $\mathrm{L}^{2}$-norm is computed, say, with a $\frac{q^{2 k} \cdot 2^{-2 i}}{256}$-precision. $\operatorname{Such} n(i, k)$ exists due to Mean Ergodic Theorem and our choice of the sets $\Delta_{g, i}^{k}$. Furthermore, it is computable, since the sequence of sets $\left(\Delta_{g, i}^{k}\right)$ is uniformly effectively open, the measure $\mu$ is computable and $\left(F_{n}\right)_{n \geq 1}$ is a computable Følner sequence.

\subsection{Birkhoff's Theorem}

In this section we prove the main theorems of the article. Our main technical tools are the generalization of Kučera's theorem from the previous section, the result of Lindenstrauss about pointwise convergence of ergodic averages and Lemmas 2.3, 2.5 about the invariance of limsup of averages. The strategy is in general similar to [2]. First, we prove Birkhoff's effective ergodic theorem for indicator functions of effectively opens sets.

Lemma 3.1 Let $\Gamma$ be a computable amenable group with a canonically computable tempered two-sided Folner sequence $\left(F_{n}\right)_{n \geq 1}$. Suppose that $\left(\{0,1\}^{\mathbb{N}}, \mu, \Gamma\right)$ is a computable ergodic Cantor system and that $U \subseteq\{0,1\}^{\mathbb{N}}$ is an effectively open set. For every Martin-Löf random $\omega \in\{0,1\}^{\mathbb{N}}$ the equality

$$
\lim _{n \rightarrow \infty} \mathbb{E}_{g \in F_{n}} \mathbf{1}_{U}(g \cdot \omega)=\mu(U)
$$

holds.

Proof First, let us show that

$$
\limsup _{n \rightarrow \infty} \mathbb{E}_{g \in F_{n}} \mathbf{1}_{U}(g \cdot \omega) \leq \mu(U)
$$

for every Martin-Löf random $\omega$. Let

$$
q>\mu(U)
$$


be some fixed rational number. Let

$$
A_{k}:=\left\{x \in\{0,1\}^{\mathbb{N}}: \sup _{n \geq k} \mathbb{E}_{g \in F_{n}} \mathbf{1}_{U}(g \cdot x)>q\right\} \quad \text { for } k \geq 1,
$$

which is an effectively open set. Pointwise ergodic theorem (Theorem 2.2) implies that $\mu\left(\bigcap_{k \geq 1} A_{k}\right)=0$, hence there is some $k \geq 1$ such that $\mu\left(A_{k}\right)<1$. Let $\omega \in\{0,1\}^{\mathbb{N}}$ be an arbitrary Martin-Löf random point. It follows from Theorem 3.1 that $\omega \notin A_{k}^{*}$, hence there exists $g_{0} \in \Gamma$ such that $g_{0} \cdot \omega \notin A_{k}$. Hence

$$
\limsup _{n \geq 1} \mathbb{E}_{g \in F_{n}} \mathbf{1}_{U}\left(g \cdot\left(g_{0} \cdot \omega\right)\right) \leq q .
$$

The function $g \mapsto \mathbf{1}_{U}(g \cdot \omega)$ on $\Gamma$ is bounded, thus we can use Lemma 2.3 to deduce that

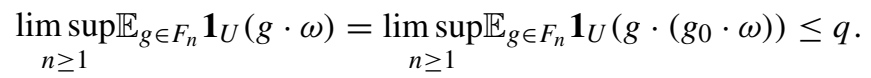

Since $q>\mu(U)$ is an arbitrary rational, this implies that $\lim \sup \mathbb{E}_{g \in F_{n}} \mathbf{1}_{U}(g \cdot \omega) \leq$ $\mu(U)$.

Secondly, if $U=\bigcup_{i \in E} I_{i}$ for an r.e. subset $E \subseteq \mathbb{N}$, we let $\Delta_{k} \subseteq U$ be the union $I_{i_{1}} \cup \cdots \cup I_{i_{k}}$ of the first $k$ intervals in $U$ for every $k \geq 1$. Then $\Delta_{k}$ is a clopen subset, and its complement $\Delta_{k}^{c}$ is an effectively open set. The preceding argument, applied to $\Delta_{k}^{c}$, implies that

$$
\underset{n \geq 1}{\limsup } \mathbb{E}_{g \in F_{n}} \mathbf{1}_{\Delta_{k}^{c}}(g \cdot \omega) \leq \mu\left(\Delta_{k}^{c}\right)=1-\mu\left(\bigcup_{j=1}^{k} I_{i_{j}}\right) .
$$

Since $k \geq 1$ is arbitrary, it follows easily that

$$
\mu(U) \leq \liminf _{n \geq 1} \mathbb{E}_{g \in F_{n}} \mathbf{1}_{U}(g \cdot \omega)
$$

and the proof is complete.

We proceed to the main theorems of the article.

Theorem 3.2 Let $\Gamma$ be a computable amenable group with a canonically computable tempered two-sided Folner sequence $\left(F_{n}\right)_{n \geq 1}$. Suppose that $\left(\{0,1\}^{\mathbb{N}}, \mu, \Gamma\right)$ is a computable ergodic $\Gamma$-system. For every bounded lower semicomputable $f$ and for every Martin-Löf random $\omega \in\{0,1\}^{\mathbb{N}}$ the equality

$$
\lim _{n \rightarrow \infty} \mathbb{E}_{g \in F_{n}} f(g \cdot \omega)=\int f d \mu
$$

holds.

Proof Firstly, the proof that

$$
\limsup _{n \rightarrow \infty} \mathbb{E}_{g \in F_{n}} f(g \cdot \omega) \leq \int f d \mu
$$


for every Martin-Löf random $\omega$ is completely analogous to the first part of the proof of Lemma 3.1 above. In particular, the argument about the translation-invariance of

$$
\limsup _{n \geq 1} \mathbb{E}_{g \in F_{n}} f(g \cdot \omega)
$$

remains valid, since $f$ is a bounded function and we can once again use Lemma 2.3.

Secondly, given an arbitrary $\varepsilon>0$, let $0 \leq h \leq f$ be a finite linear combination of indicator functions of effectively open sets such that

$$
\|f-h\|_{1} \leq \varepsilon .
$$

An application of Lemma 3.1 yields that

$$
\liminf _{n \geq 1} \mathbb{E}_{g \in F_{n}} f(g \cdot \omega) \geq \liminf _{n \geq 1} \mathbb{E}_{g \in F_{n}} h(g \cdot \omega) \geq \int h d \mu \geq \int f d \mu-\varepsilon,
$$

which completes the proof, since $\varepsilon>0$ is arbitrary.

Remark 3.1 Compared to [2], we make an additional assumption in Theorem 3.2 that the observable is bounded. The reason for that is that the invariance of lim sup is only in general guaranteed by Lemma 2.3 for bounded functions.

In a special case, when $\Gamma$ is a computable group of polynomial growth, we can remove the additional assumption about the boundedness of $f$. The theorem below is a generalization of [2, Theorem 8$]$.

Theorem 3.3 Let $\Gamma$ be a computable group of polynomial growth with the Folner sequence of balls around $\mathrm{e} \in \Gamma$ given by

$$
F_{n}:=\{g \in \Gamma:\|g\| \leq n\} \quad \text { for } n \geq 1 .
$$

Suppose that $\left(\{0,1\}^{\mathbb{N}}, \mu, \Gamma\right)$ is a computable ergodic $\Gamma$-system. For every lower semicomputable $f$ and for every Martin-Löf random $\omega \in\{0,1\}^{\mathbb{N}}$ the equality

$$
\lim _{n \rightarrow \infty} \mathbb{E}_{g \in F_{n}} f(g \cdot \omega)=\int f d \mu
$$

holds.

Proof The argument is identical to the reasoning in Theorem 3.2. We use Lemma 2.5 for the invariance of lim sup of averages, hence obtaining the proof for an arbitrary lower semicomputable $f$.

Acknowledgements This research was carried out during the author's $\mathrm{PhD}$ studies in Delft University of Technology under the supervision of Markus Haase. I would like to thank him for his support. The author also kindly acknowledges the financial support from Delft Institute of Applied Mathematics. The author would also like to thank anonymous referees for the careful reading and many helpful suggestions. 
Open Access This article is distributed under the terms of the Creative Commons Attribution 4.0 International License (http://creativecommons.org/licenses/by/4.0/), which permits unrestricted use, distribution, and reproduction in any medium, provided you give appropriate credit to the original author(s) and the source, provide a link to the Creative Commons license, and indicate if changes were made.

\section{References}

1. Bass, H.: The degree of polynomial growth of finitely generated nilpotent groups. Proc. London Math. Soc. (3) 25, 603-614 (1972)

2. Bienvenu, L., Day, A.R., Hoyrup, M., Mezhirov, I., Shen, A.: A constructive version of Birkhoff's ergodic theorem for Martin-Löf random points. Inf. Comput. 210, 21-30 (2012). https://doi.org/10.1016/j.ic.2011.10.006

3. Ceccherini-Silberstein, T., Coornaert, M.: Cellular Automata and Groups. Springer Monographs in Mathematics. Springer, Berlin (2010). https://doi.org/10.1007/978-3-642-14034-1

4. Eisner, T., Farkas, B., Haase, M., Nagel, R.: Operator Theoretic Aspects of Ergodic Theory. Springer, Cham (2015). https://doi.org/10.1007/978-3-319-16898-2

5. Galatolo, S., Hoyrup, M., Rojas, C.: Effective symbolic dynamics, random points, statistical behavior, complexity and entropy. Inf. Comput. 208(1), 23-41 (2010). https://doi.org/10.1016/j.ic.2009.05.001

6. Glasner, E.: Ergodic Theory via Joinings, Mathematical Surveys and Monographs, vol. 101. American Mathematical Society, Providence (2003). https://doi.org/10.1090/surv/101

7. Gromov, M.: Groups of polynomial growth and expanding maps. Inst. Hautes Études Sci. Publ. Math. 53, 53-73 (1981). https://mathscinet.ams.org/mathscinet-getitem?mr=623534

8. Hoyrup, M., Rojas, C.: Computability of probability measures and Martin-Löf randomness over metric spaces. Inf. Comput. 207(7), 830-847 (2009). https://doi.org/10.1016/j.ic.2008.12.009

9. Lindenstrauss, E.: Pointwise theorems for amenable groups. Invent. Math. 146(2), 259-295 (2001). https://doi.org/10.1007/s002220100162

10. Ornstein, D.S., Weiss, B.: Entropy and isomorphism theorems for actions of amenable groups. J. Analyse Math. 48, 1-141 (1987). https://doi.org/10.1007/BF02790325

11. Pansu, P.: Croissance des boules et des géodésiques fermées dans les nilvariétés. Ergodic Theory Dynam. Syst. 3(3), 415-445 (1983). https://doi.org/10.1017/S0143385700002054

12. Rabin, M.O.: Computable algebra, general theory and theory of computable fields. Trans. Amer. Math. Soc. 95, 341-360 (1960)

13. Shen, A., Vereshchagin, N.: Computable Functions. Transl. from the Russian By V. N. Dubrovskii. American Mathematical Society (AMS), Providence (2003)

14. V'yugin, V.: Effective convergence in probability and an ergodic theorem for individual random sequences. Teor. Veroyatn. Primen. 42(1), 35-50 (1997). https://doi.org/10.4213/tvp1710 